\title{
Piecemeal or big bangs: correlating the vertebrate evolution with proposed models of gene expansion events
}

\section{Amir Ali Abbasi}

In their Opinion article (The evolutionary significance of ancient genome duplications. Nature Rev. Genet. 10, 725-732 (2009)) $)^{1}$, Van de Peer et al. attempt to correlate species diversification, morphological transitions and the appearance of evolutionary novelties with ancient whole-genome duplications (WGDs) in plants, vertebrates, budding yeast and unicellular ciliate protozoa. However, the arguments presented might not be relevant to vertebrates. Despite extensive empirical scrutiny, the occurrence of two rounds of WGD (the $2 \mathrm{R}$ hypothesis) is controversial ${ }^{2-5}$. Therefore, establishing a link between vertebrate evolutionary diversification and proposed WGDs must require enough evidence to falsify the null hypothesis, namely that the vertebrate genome evolved through piecemeal duplications that occurred at different time points during animal history ${ }^{2,3,6,7}$. In fact, all of the events that Van de Peer and colleagues correlate with polyploidy can be explained under this 'small-scale duplication' model of animal genome evolution.

Arguments in support of $2 \mathrm{R}$ are based on: higher numbers of genes in vertebrates than in invertebrates; the occurrence of conserved syntenic fragments in vertebrate genomes; rapid expansion of multigene families during early vertebrate history; and the extrapolation of genome evolution scenarios in plants and yeast to genome evolution events in vertebrates ${ }^{8-10}$. However, many studies of $2 \mathrm{R}$ are based on data from few vertebrates and from highly derived invertebrate genomes and therefore generate a distorted picture of the early history of the vertebrate genome. The recent availability of genomic data from an expanding range of vertebrate and invertebrate species from interspersed time points ${ }^{2}$ reveals that the genomes of morphologically simple animals, such as sea anemones and sea urchins, are unexpectedly complex in terms of gene content and structure. This suggests that core components of the vertebrate genetic toolkit originated and diversified in the ancestral deuterostome genome or even in the common ancestor of bilateriannonbilaterian animals, and were subsequently contracted specifically in the genomes of model protostomes (for example, fruitflies) and non-vertebrate chordates (for example, amphioxi) ${ }^{11,12}$. These observations support the view that there is no simple relationship between gene number and organismal complexity. Instead, a growing body of evidence suggests that organismal complexity and diversity is correlated with redeploying ancestral gene sets to different tissues and developmental stages during evolution.

A more realistic model of ancient vertebrate genome history can be deduced by tracing the evolutionary trajectory of the genomes of recently diverged vertebrate species. For instance, the human genome contains a complex pattern of recent segmental duplications (SDs); large blocks of genomic sequence (300 kb-1 Mb) that are present in at least two locations and that show high identity $(>90 \%)^{13}$. Several roles have been attributed to these SDs, including the creation of new genes, the expansion of multigene families and the triggering of large-scale chromosomal rearrangements in hominoids ${ }^{14}$. These findings support the notion that small-scale duplications and rearrangements might have been a predominant mechanism in shaping the vertebrate genome throughout evolutionary history ${ }^{2}$.

I would argue that there is currently no conclusive evidence to support the occurrence of $2 \mathrm{R}$ at the root of vertebrate lineage; therefore it is not prudent to infer correlations between this unproven event and vertebrate evolutionary diversity.

Amir Ali Abbasi is at the National Center for Bioinformatics, Faculty of Biological Sciences, Quaid-i-Azam University, Islamabad 45320, Pakistan e-mail:abbasiam@qau.edu.pk doi: 10.1038/nrg2600-c1

1. Van de Peer, Y., Maere, S. \& Meyer, A. The evolutionary significance of ancient genome duplications. Nature Rev. Genet. 10, 725-732 (2009). 2. Abbasi, A. A. Are we degenerate tetraploids? More genomes, new facts. Biol. Direct 3, 50 (2008).

3. Abbasi, A. A. \& Grzeschik, K.-H. An insight into the phylogenetic history of $H O X$ linked gene families in vertebrates. BMC Evol. Biol. 7, 239 (2007).

4. Donoghue, P. C. \& Purnell, M. A. Genome duplication extinction and vertebrate evolution. Trends Ecol. Evol. 20, 312-319 (2005).

5. Furlong, R. F. $\&$ Holland, P. W. Polyploidy in vertebrate ancestry: Ohno and beyond. Biol. J. Linn. Soc. Lond. 82, 425-430 (2004).

6. Hughes, A. L. \& Friedman, R. 2R or not 2R: testing hypotheses of genome duplication in early vertebrates. J. Struct. Funct. Genomics 3, 85-93 (2003).

7. Martin, A. P. Increasing genomic complexity by gene duplication and the origin of vertebrates. Am. Nat. 154, 111-128 (1999).

8. Dehal, P. \& Boore, J. L. Two rounds of whole genome duplication in the ancestral vertebrate. PLOS Biol. 3, e314 (2005).

9. McLysaght, A., Hokamp, K. \& Wolfe, K. H. Extensive genomic duplication during early chordate evolution. Nature Genet. 31, 200-204 (2002).

10. Sundstrom, G., Larsson, T. A. \& Larhammar, D. Phylogenetic and chromosomal analyses of multiple gene families syntenic with vertebrate Hox clusters. BMC Evol. Biol. 8, 254 (2009).

11. Putnam, N. H. et al. Sea anemone genome reveals ancestral eumetazoan gene repertoire and genomic organization. Science 317, 86-94 (2007).

12. Sodergren, E. et al. The genome of the sea urchin Strongylocentrotus purpuratus. Science $\mathbf{3 1 4}$ 941-952 (2006).

13. Bailey, J. A. \& Eichler, E. E. Primate segmental duplications: crucibles of evolution, diversity and disease. Nature Rev. Genet. 7, 552-564 (2006).

14. Marques-Bonet, T., Girirajan, S. \& Eichler, E. E. The origins and impact of primate segmental duplications. Trends Genet. 25, 443-454 (2009).

\section{Acknowledgements}

This work was supported by the National Center for Bioinformatics, Quaid-i-Azam University, Islamabad. 